\title{
PRONUNCIATION ERROR DETECTION FOR COMPUTER ASSISTED PRONUNCIATION TEACHING IN MANDARIN
}

\author{
Min-Siong Liang ${ }^{1}$, Jian-Yung Hung ${ }^{2}$, Ren-Yuan Lyu ${ }^{3} *$, Yuang-Chin Chiang ${ }^{4}$ \\ ${ }^{1}$ Network and Multimedia Institute, Institute for Information Industry \\ ${ }^{2}$ Advanced Technology Center, Industrial Technology Research Institute, Hsinchu \\ ${ }^{3}$ Inst. of Computer Science and Information Engineering, Taoyuan \\ ${ }^{4}$ Inst. of Statistics, National Tsing Hua University, Hsinchu \\ *E-mail: renyuan.lyu@gmail.com
}

\begin{abstract}
In this paper, we provided a strategy of error detection of pronunciation and applied it to the computer-assisted pronunciation teaching(CAPT), especially in Mandarin language learning. In our system, it can be divided into two parts: the sentence verification(SV) and syllable identification(SI). First was used to ban out-of-task sentences. We used the likelihood ratio test, which was computed between the maximum probability of a result under two different hypotheses, i.e. null hypothesis and alternative hypothesis models, to verify the deviation degree and decide whether the student pronunciation is out-of-task. In SV part, the experimental results was significant and had $91.0 \%$ rate of F-score. The second part was applied to recognize the content of speech read by the speaker. The recognition net was built as a sausage shape with pronunciation confusion table corresponding to confusion error patterns. Then, the system could find out the wrong pronounced syllable for the appropriate feedback to correct the pronunciation of the users. In the stage of SI, the best detection rate had a F-score rate of $77.2 \%$.
\end{abstract}

Index Terms - computer assisted language teaching (CAPT), pronunciation error detection, sentence verification, syllable identification, Mandarin

\section{INTRODUCTION}

This paper describes an approach to pronunciation error detection for Computer-Assisted Pronunciation Teaching (CAPT). For CAPT, many researchers have developed it using speech recognition and synthesis techniques $[1,2]$. So far, most approaches for CAPT used the Hidden Markov Models (HMM) log-likelihood-based algorithm score [3, 4], but few could detect and verify error for users. However, the report by Neri et al. [5] claimed that the effectiveness of learning depended on the corrective feedback of a CAPT system, which needed a precise pronunciation error detector. In addition, the pronunciation errors hypotheses often used linguistic knowledge, which was often language-dependent and derived by more than one linguists $[6,7]$, but the linguistic knowledge was sometimes contradictory with each other. In this paper, therefore, we try to integrate data-driven based and knowledge-based methods for generation of pronunciation errors hypotheses to propose a new framework for CAPT in Mandarin.

As Fig. 1 was shown, our goal was to filter out the outof-task sentences in the first stage and recognize the confusion or target pronunciations in the next stage. Therefore, the pronunciation error detection can be divided into two parts: sentence verification and syllable identification. In the sentence verification part, we were trying to ban out-of-task sentences with likelihood ratio test by comparing the correct pronunciation model and anti-pronunciation model. In order to give the corrective feedback with the right syllable or word in the next stage, we hope that the system could track considerable misreading, miscue, even the out-of-task sentences more accurately. On the other hand, the system was expected to recognize the speaker's pronunciation in phoneme or syllable level in the syllable identification part. The proposed solution to this problem is to expand the searching net with pronunciation variation (PV) rules. Even native speakers would have a pronunciation variation (PV) due to individual habits and accents. Collecting the PV patterns as many as possible might be a better way to find appropriate PV rules. However, how to rank those collected rules became another important issue. Therefore, we not only collect variation patterns but incorporated the linguistic knowledge and the ranking method to build the pronunciation searching network.

In Taiwan, Mandarin is one of three major languages (Mandarin, Taiwanese and Hakka) and is widely used as the native tongue. So far, for second language pronunciation learning, most systems were developed in English and few were built in Mandarin. Therefore, we were eager to build a language learning system in Mandarin and develop a prototype system. 


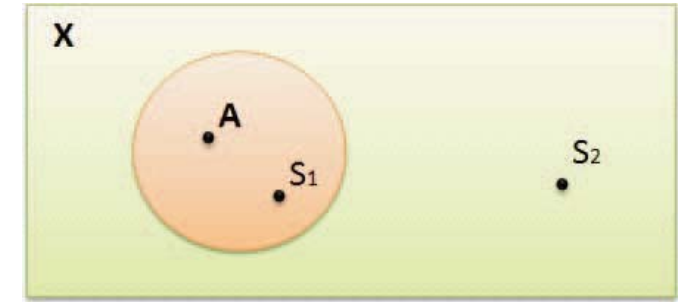

Fig. 1. The categories of pronunciation, where $X, A, S_{1}$ and $S_{2}$ can be represented as all possible pronunciations, target pronunciation, confusion pronunciation and out-of-task pronunciation respectively.

\section{THE SENTENCE VERIFICATION FOR CAPT}

To enhance error detection, the HMM-based sentence verification was used to filter out out-of-task pronunciations by likelihood ratio test. Given input speech to an HMM recognizer, let $P_{k}$ be the most likely string of syllables by Viterbi decoding. $P_{k}$ can be a concatenation of phoneme units which can be written as

$$
P_{k}=p_{1}^{(k)} p_{2}^{(k)} \ldots p_{N_{k}}^{(k)}
$$

where the phoneme string $p_{1}^{(k)} p_{2}^{(k)} \ldots p_{N_{k}}^{(k)}$ is the phoneme phoneme lexical representation of $P_{k}$, and $N_{k}$ is the number of phoneme units comprising $P_{k}$. The log-likelihood ratio test can be written as follows:

$\log \frac{P\left(O \mid H_{0}\left(P_{k}\right)\right)}{P\left(O \mid H_{1}\left(P_{k}\right)\right)}=\log P\left(O \mid H_{0}\left(P_{k}\right)\right)-\log P\left(O \mid H_{1}\left(P_{k}\right)\right)$

where $P\left(O \mid H_{0}\left(P_{k}\right)\right)$ and $P\left(O \mid H_{1}\left(P_{k}\right)\right)$ were the likelihoods of the observation sequence $O$ given the null hypothesis that $P_{k}$ was spoken and the alternate hypothesis that $P_{k}$ was not spoken respectively.

In order to find the better models for $H_{0}$ and $H_{1}$, we built four kinds of models, including Tri-phone models, garbage model(GM), pronunciation manner cluster(PMC) models and anti-PMC models. Table 1 showed how we clustered the Mandarin phonemes for PMC models. Instead, the anti-PMC models were trained by the whole observations except those of the phonemes of the same PMC cluster. Also, the GM model was trained by all observations of the whole phonemes.

\subsection{Evaluation of the Sentence Verification}

For the four kinds of acoustic models mentioned above, we chose speaker-independent HMM models trained by Formosa Speech Database (ForSDAT) [8]. The features were extracted into vectors of 48 dimensional MFCC plus 4 dimensional energy. For testing data collection, five sentences with 25 syllables were designed to cover all phonemes in Mandarin. Then, according to the table 1 , we replaced the five sentences
Table 1. The pronunciation manner cluster (PMC) transcribed in IPA in Mandarin.

\begin{tabular}{llll}
\hline Consonant Class & Phone & Vowel Class & Phone \\
\hline CS & b, b', t, t', k, k' & VI & i, y \\
CA & ts, ts', ts, ts' & VA & $\mathrm{a}$ \\
CF & f, s, s, z, x & VU & $\mathrm{u}$ \\
CN & m, n, s & VO & o \\
CL & 1 & VER & $r, \partial^{\circ}$ \\
& & VE & $\varepsilon$ \\
\hline
\end{tabular}

Table 2. The distribution of out-of-task(OT) and confusion $(C F)$ syllables in the two kinds of prompt sheets. Num. and sen. syl. are denoted as number, sentence and syllable. "Original:OT" means how many original and out-of-task syllables was in one sentence respectively.

\begin{tabular}{ll|ll}
\hline Original:OT & Num. of sen. & Original:CF & Num. of sen. \\
\hline $5: 0$ & 5 & $5: 0$ & 5 \\
$4: 1$ & 25 & $4: 1$ & 20 \\
$3: 2$ & 50 & $3: 2$ & 25 \\
$2: 3$ & 50 & $2: 3$ & 25 \\
$1: 4$ & 25 & $1: 4$ & 20 \\
$0: 5$ & 5 & $0: 5$ & 5 \\
\hline
\end{tabular}

with out-of-task(OT) or confusion(CF) syllables to form 160sentence and 100-sentence prompt sheets for out-of-task and confusion pronunciation testing. All phonemes for each outof-task syllable were chosen in the different clusters corresponding the original syllable. However, in order to form one confusion syllable, only one of phonemes of the original syllable would be replaced and chosen in the same cluster. For example, /bai/ can be the confusion pronunciation of /b'ai/ whereas the pronunciation of /xuan/ was out-of-task. The distribution of these two kinds of prompt sheet was shown as Table 2. Four native speakers were required to record the two prompt sheets and the statistics of the corpus was shown in Table 3. Then, the criterion of rejection for a sentence occurred where a half of syllables was out-of-task. Finally, the experiment results were displayed in Fig. 2. In addition, we also adopted F-measure to represent the performance of these acoustic models and found that the pair of tri-phone model versus anti-pmc was the best of them shown in Table 4. Therefore, we decided the output of tri-phone vs. anti-PMC model as the input of testing data of the next stage, i.e. syllable identification. 
Table 3. The statistics of the testing corpus. OT, CF, Sen. syl. and num. are denoted as out-of-task, confusion, sentence, syllable and number.

\begin{tabular}{l|lll}
\hline & Num. of sen. & Num. of syl. & Time \\
\hline OT corpus & 640 & 3200 & 23 mins \\
CF corpus & 400 & 2000 & 20 mins \\
\hline
\end{tabular}

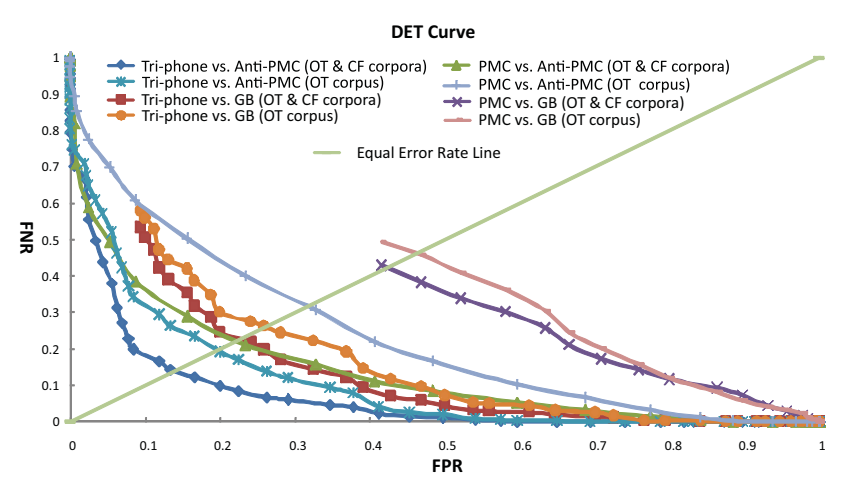

Fig. 2. The experiment results of sentence verification under the OT and CF corpora with the combinations of Tri-phone, PMC, and GM, Anti-PMC models.

\section{THE SYLLABLE IDENTIFICATION FOR CAPT}

\subsection{Confusion Table Construction for Deriving PV Rules}

The same simple way to adopt the methodology of pronunciation variation is to expand the pronunciation lexicon using variation rules of the form "LBR $\rightarrow$ LSR", where B and S represent the base form and surface form of a central phone, and $\mathrm{L}, \mathrm{R}$ are the left and right contexts respectively. To derive such rules, a speech corpus with both canonical pronunciation and actual pronunciation is necessary, which a similar work was proposed in [9]. It could be shown that as long as the pronunciation rules could be well chosen, the phonetic detection performance would be effectively improved.

We choose a subset of ForSDAT, called ForSDAT-02, to derive PV rules and the statistical information was summarized as in Table 5. ForSDAT-02 is a speech database with rich bi-phone coverage. The speech is recorded by first prompting

Table 4. The best performance for the combination of PMC, Anti-PMC, GM and Tri-phone models under the OT and CF corpora.

\begin{tabular}{llll}
\hline & Precision & Recall & F-Score \\
\hline PMC vs Anti-PMC & $81.0 \%$ & $91.7 \%$ & $86 \%$ \\
PMC vs GM & $69 \%$ & $100 \%$ & $81.9 \%$ \\
Tri-phone vs Anti-PMC & $88.9 \%$ & $93.2 \%$ & $91.0 \%$ \\
Tri-phone vs GM & $81.1 \%$ & $95.6 \%$ & $87.8 \%$ \\
\hline
\end{tabular}

a transcript to the speakers. A small portion of the speech data about 1600 utterances was then manually checked and the phonetic transcription of the transcript "corrected" according to actual speech. Some examples of the original transcription (the base-form) and the manually corrected transcription (the surface-form) are shown in Table 6, which is called the tri-phone level table.

Table 5. The statistics of ForSDAT-01 speech corpus and partially manually validated ForSDAT-02 speech corpus (M: male, F: female, Tri.:triphones).

\begin{tabular}{lll}
\hline & ForSDAT-01 & Partial ForSDAT-02 \\
\hline Utterance & 92158 & 19731 \\
\# of People & 100(M: 50, F:50) & $131(\mathrm{M}:$ 72, F:59) \\
\# of Syllables & 179730 & 45865 \\
\# of distinct tri. & 1356 & 1194 \\
\# of total tri. & 555731 & 104894 \\
Time(hr) & 22.43 & 7.2 \\
\hline
\end{tabular}

Table 6. Triphone-level confusion table, where $n_{i j}$ represents the number of variation from triphone $b_{i}$ to triphone $s_{j}, P$ is the number of surface-form and base-form, $N_{i}=\sum_{j} n_{i j}$, $M_{j}=\sum_{i} n_{i j}$ and $N=\sum_{i} \sum_{j} n_{i j}$.

\begin{tabular}{l|lllllll|l}
\hline & b- $\gamma$ & i-n & i-n & $\ldots$ & $s_{j}$ & $\ldots$ & b-o & \\
\hline b- $\gamma$ & 237 & 0 & 0 & $\ldots$ & $n_{1 i}$ & $\ldots$ & 30 & 267 \\
i-n & 0 & 1273 & 84 & $\ldots$ & $n_{2 i}$ & $\ldots$ & 0 & 1373 \\
$\vdots$ & $\vdots$ & $\vdots$ & $\vdots$ & $\vdots$ & $\vdots$ & $\vdots$ & $\vdots$ & $\vdots$ \\
$b_{i}$ & $n_{i 1}$ & $n_{i 2}$ & $n_{i 3}$ & $\ldots$ & $n_{i j}$ & $\ldots$ & $n_{i, P}$ & $N_{i}$ \\
$\vdots$ & $\vdots$ & $\vdots$ & $\vdots$ & $\vdots$ & $\vdots$ & $\vdots$ & $\vdots$ & $\vdots$ \\
a-m & 0 & 0 & 0 & $\ldots$ & $n_{P j}$ & $\ldots$ & 0 & 869 \\
\hline & 241 & 1315 & 1102 & $\ldots$ & $M_{j}$ & $\ldots$ & 107 & $N$ \\
\hline
\end{tabular}

\subsection{Ranking PV Rules with Data-driven based method and incorporating knowledge-based rules}

First of all, the criterion should be adopted to choose the most significant rule sets. It is mutual information(MI) of the base form pronunciation and the surface form pronunciation. The mathematic definitions of the above measure is as follow:

Mutual information of the base form pronunciation $b_{i}$, and the surface form pronunciation $s_{j}$,

$I_{i j}=p\left(b_{i}, s_{j}\right) \log \frac{p\left(b_{i}, s_{j}\right)}{p\left(b_{i}\right) p\left(s_{j}\right)}=\frac{n_{i j}}{N} \log \left(N \cdot \frac{n_{i j}}{\sum_{i} n_{i j} \cdot \sum_{j} n_{i j}}\right)$

In all the above equation, $n_{i j}$ is the number of (base-form) triphone $b_{i}$ substitutions by the surface-form triphone $s_{j}$ that appear in a corpus, and $N=\sum_{i} \sum_{j} n_{i j}, N_{i}=\sum_{j} n_{i j}$, 
$p\left(b_{i}, s_{j}\right)$ represents the joint probability of $\left(b_{i}, s_{j}\right)$,

$p\left(b_{i}\right)$ and $p\left(s_{j}\right)$ equal the marginal probability of $b_{i}$ and $s_{j}$, respectively. Note that each pair $(i, j), i \neq j$, corresponds to a substitution rule and we select those pairs $(i, j)$ with higher scores of $p\left(b_{i}, s_{j}\right), p\left(b_{i}, s_{j}\right)$ and $I_{i j}$ to be the variation rules to extend the sausage net pronunciation.

In addition, the variation rules also incorporated the linguistic knowledge [10]. The list of knowledge-based rules was shown in Table 7. In Finally, in this stage, we also use F-measure (F-score) to evaluate our system under OT and CF corpora. The performance of syllable identification was presented in Table 8.

Table 7. The list for Knowledge-based rules including oneway and two-way patterns.

\begin{tabular}{ll}
\hline One-way Pattern & Two-way Pattern \\
\hline $\mathrm{z} \rightarrow \mathrm{Z}$ & $\mathrm{b} \leftrightarrow \mathrm{b}^{\prime}$ \\
$\mathrm{s} \rightarrow \mathrm{s}$ & $\mathrm{b} \leftrightarrow \mathrm{t}$ \\
$\mathrm{t} \mathrm{s} \rightarrow \mathrm{ts}$ & $\mathrm{b} \leftrightarrow \mathrm{t}^{\prime}$ \\
$\mathrm{t} \mathrm{s}^{\prime} \rightarrow \mathrm{ts}$, & $\mathrm{t} \leftrightarrow \mathrm{t}^{\prime}$ \\
$\mathrm{y} \rightarrow \mathrm{i}$ & $\mathrm{t}^{\prime} \leftrightarrow \mathrm{k}^{\prime}$ \\
& $\mathrm{k} \leftrightarrow \mathrm{k}^{\prime}$ \\
& $\mathrm{n} \leftrightarrow \mathrm{l}$ \\
\hline
\end{tabular}

Table 8. The best performance for Mutual-information-based and Knowledge-based measure under the OT and CF corpora.

\begin{tabular}{llll}
\hline & Precision & Recall & F-Score \\
\hline Mutual-Information & $66.7 \%$ & $91.5 \%$ & $77.2 \%$ \\
\hline
\end{tabular}

\section{DISCUSSION AND CONCLUSIONS}

We have proposed a new approach to address pronunciation error detection for Computer-Assisted Pronunciation Teaching (CAPT). In our system, it can be divided into two parts: the sentence verification and syllable identification. To enhance error detection, we were trying four kinds of combinations to find the more better performance to filter out out-oftask pronunciations by likelihood ratio test. In this stage, the experimental results under OT and CF corpora were significant and has $91.0 \%$ rate of F-score in combination of Triphone vs. Anti-PMC (anit- pronunciation manner cluster) models.

In pronunciation variation rules (PV-rules) selection, the data-driven variation rules, which were derived using mutual information(MI) measure, were used to extend more possible pronunciations. In addition to the data-driven measure, the linguistic knowledge was also integrated into the PV-rules. In the stage of syllable identification, the best detection rate had a F-score rate of $77.2 \%$, which was the best among the other measures in this paper. Finally, we used these two components to build a prototype of CAPT as shown in Fig. 1.

In the future, the more suitable acoustic models were still an unsolved problem in this research. The possible method might be to adapt the common acoustic models by the analysis of probability density function between mother-tongue and the second languages. In addition, the complex tone sandhi should also be accompanied with tone recognition.

\section{REFERENCES}

[1] M.-S. Liang, et al., "A Taiwanese Text-to-Speech System with Applications to Language Learning", In Proc. ICALT 2004, Joensuu, Finland, 2004.

[2] Abdou, S. M., et al.,"Computer Aided Pronunciation Learning System Using Speech Recognition Techniques", In Proc. Interspeech 2006, Pittsburgh, Pennsylvania, 2006.

[3] Witt, S., et al.,"Language Learning Based on NonNative Speech Recognition”, In Proc. Eurospeech 97, Rhodes, Greece, September 22-25, 1997.

[4] Wei, S., et al., "Automatic Mandarin Pronunciation Scoring for Native Learners with Dialect Accent", In Proc. Interspeech 2006, Pittsburgh, Pennsylvania, 2006.

[5] Neri, A., et al., "ASR-based Corrective Feedback on Pronunciation: does it really work?", In Proc. Interspeech 2006, Pittsburgh, Pennsylvania, 2006.

[6] Tsubota, Y., et al.,"Recognition and Verification of English by Japanese Students for Computer-Assisted Language Learning Sytem", In Proc. ICSLP 2002, Denver, USA, 2002.

[7] Chen, J.-C., et al.,"Formant-Based English Vowel Assessment for Chinese in Taiwan", In Proc. Interspeech 2006, Pittsburgh, Pennsylvania, 2006.

[8] Lyu, R.-Y., et al.,"Toward Constructing A Multilingual Speech Corpus for Taiwanese (Minnan), Hakka, and Mandarin", International Journal of Computational Linguistics and Chinese Language Processing (IJCLCLP), Vol. 9, No. 2, August 2004, pp. 1-12.

[9] M.-S. Liang, et al., "Phonetic Transcription using Speech Recognition Technique Considering Variations in Pronunciation," In: Proc. IEEE Int. Conf. Acoustics, Speech, and Signal Processing (ICASSP), 2007.

[10] Lyu, R.-Y., et al.,"Advances in Chinese Spoken Language Processing", World Scientific, Chapter 17, pp.387-406, 2007. 